\title{
Relations à distance des migrants et réseaux d'insertion à Dakar
}

\section{Abdou Salam Fall}

\section{OpenEdition}

Journals

Édition électronique

URL : http://journals.openedition.org/apad/3213

ISSN : 1950-6929

Éditeur

LIT Verlag

Édition imprimée

Date de publication : 1 juin 1993

Référence électronique

Abdou Salam Fall, «Relations à distance des migrants et réseaux d'insertion à Dakar », Bulletin de I'APAD [En ligne], 5 | 1993, mis en ligne le 04 juin 2008, consulté le 07 septembre 2020. URL : http:// journals.openedition.org/apad/3213

Ce document a été généré automatiquement le 7 septembre 2020

Bulletin de l'APAD 


\title{
Relations à distance des migrants et réseaux d'insertion à Dakar ${ }^{1}$
}

\author{
Abdou Salam Fall
}

1 Le maintien de forts courants migratoires du milieu rural vers les villes et surtout en direction de la grande agglomération de Dakar compte parmi les problèmes cruciaux du Sénégal. Cette permanence des flux migratoires vers l'agglomération de Dakar entraîne une explosion urbaine devenue préoccupante.

2 Les déplacements obéissent à des motivations à la fois individuelles et collectives et sont intrinsèquement liés aux déséquilibres affectant les zones de départ. Migrer signifie élargir son espace de vie, aller à la recherche des moyens de production et de survie. C'est donc s'investir ailleurs temporairement, périodiquement ou durablement. Par la migration ce sont les forces productives qui se délocalisent et se redisposent dans un espace élargi dépassant le simple milieu d'origine. Plus qu'un mouvement d'acteurs sociaux individuellement considérés, ce sont des groupes ou communautés qui se forment mettant ainsi en rapport des unités économiques "déspatialisées".

3 Par des réseaux sociaux multiformes, les migrants maintiennent des relations à distance plus ou moins permanentes, plus ou moins occasionnelles avec leur localité d'origine. Plusieurs raisons expliquent cette stratégie. Dans certaines localités où la crise de l'agriculture est la plus prononcée, la dépendance de la production et des budgets des ménages des apports attendus des migrants en ville semble irréversible. C'est ce que nous avons relevé en pays sereer auprès de chefs de famille considérant "qu'une maison qui ne compte aucun émigré ne peut vivre décemment (Fall, 1992)".

4 Migrer, c'est le plus souvent créer de nouveaux liens sans que cela n'induise la rupture d'anciennes relations structurantes ou fonctionnelles. Les raisons évoquées par Pontié et Lericollais (1991) sont illustratives de cette logique relationnelle de nombreuses sociétés rurales : "La nature des relations sociales, religieuses mais aussi économiques entre migrants et société d'origine est largement conditionnée par la possibilité qui leur est offerte de retourner éventuellement cultiver au pays, en cas d'échec dans son entreprise migratoire, à la "retraite", ou encore si la situation familiale - mort d'un aîné par exemple - le lui commande. La possibilité d'accès à quelques parcelles, même après 
une très longue absence, est pour le migrant la marque de reconnaissance de sa citoyenneté villageoise et lignagère autant qu'une nécessité économique".

Nous aborderons ici les relations à distance des migrants vis-à-vis de leur milieu d'origine sous le prisme des réseaux d'insertion urbaine. Une des manières de saisir le recours à un réseau est de déterminer à quelle personne l'enquêté a fait appel pour trouver un travail, pour obtenir un logement, pour maintenir ou non des liens avec son milieu d'origine. Le réseau social désigne un tissu complexe de rapports sociaux qui apparaissent sous la forme de circuits d'accueil et/ou d'insertion socio-professionnelle, résidentielle, de solidarité humaine, de relations privilégiées. Le réseau est identifié à un relais social, mais un relais qui serait construit parallèlement aux appareils et institutions sociales et quelques fois opposés à eux (Fall, 1991a). En partant du recours éventuel aux réseaux d'insertion urbaine pour situer l'effectivité des relations à distance des migrants vis-à-vis de leur lieu de provenance, nous avons fait le choix de privilégier une lecture du phénomène migratoire à partir de la ville. Nous sommes intéressés à mesurer la persistance ou le développement de liens à distance entretenus de la ville, en les comparant avec l'importance de nouvelles relations sociales rendues nécessaires par les formes actuelles d'urbanité.

Éléments de méthode

6 Nombreuses sont les méthodes utilisées pour analyser l'impact des migrations aussi bien sur les zones de départ que de destination. Selon le tableau dressé par Pontié et Lericollais (1991) les méthodes les plus pertinentes tentent de mesurer les effets des migrations par une approche globale et interactive à partir du lieu d'observation. Ainsi, dans une étude consacrée aux migrants Sereer (Sénégal), ces auteurs ont privilégié une combinaison de trois types d'approche se réalisant sur un même terrain : l'approche socio-anthropologique fondée sur une étude qualitative intensive d'un nombre restreint et significatif d'unités socio-économiques en partant d'une reconstitution des liens d'ordre généalogique afin de passer en revue chacun des membres d'un lignage tout en s'informant sur leur localisation actuelle et leur apport éventuel dans leur unité familiale; le contrôle des mouvements migratoires par des données démographiques collectées sur une longue durée; les méthodes de mesure et de suivi pratiquées en géographie agraire notamment le contrôle par le parcellaire, les techniques culturales et la main d'œuvre mobilisée, la provenance et le niveau de l'équipement agricole dans les segments de patrilignage considérés comme l'unité fonctionnelle de gestion de la terre. Ce procédé particulièrement riche et fiable demande cependant une présence prolongée et renouvelée de l'équipe pluridisciplinaire sur un terrain qui ne saurait être vaste. Une telle monographie permet de relever la pertinence de l'interaction entre différents phénomènes et disciplines, le croisement de différents outils de collecte et d'analyse. Elle ne peut s'appliquer qu'à un point d'observation situé en milieu rural. Elle permet de mettre en relief tout ou presque l'espace de vie des acteurs et groupes "déspatialisés" dont le milieu d'origine est à l'étude. Elle est donc révélatrice des relations à distance complexes entretenues par les migrants vis-à-vis de leur milieu d'origine.

7 Pour notre part, en partant de la ville comme point d'observation, nous avons préconisé une méthode à la fois quantitative et qualitative d'analyse de l'impact des migrations. Grâce à un questionnaire biographique quantitatif comme instrument de mesure des modalités de l'insertion urbaine, nous avons interrogé les migrants vivant dans différents quartiers de l'agglomération de Dakar selon une base de sondage 
définie pour toute l'enquête. Quatre types de questions concernent les relations à distance. Les visites effectuées par les migrants, les biens possédés avant la migration avec possibilité de les comparer avec ceux possédés au moment de l'enquête (cet aspect ne sera pas abordé dans cet article faute d'espace) et enfin les possibilités de recours aux réseaux d'origine notamment par le moyen de personnes localisées dans le milieu d'émigration pour l'accès à l'emploi ou au logement à Dakar. Afin d'approfondir ces données, un sous-échantillon est sélectionné selon le principe du choix raisonné et soumis à des entretiens-récits de vie (Fall, 1991c). Ce va et vient du quantitatif au qualitatif permet de combiner la représentativité et l'exemplarité.

8 Ces deux approches sont évidemment complémentaires. Elles ont en commun de partir de l'individu pour ensuite saisir le groupe social dans une démarche à la fois exploratoire et approfondie. Elles privilégient les stratégies mises en œuvre par les individus et les groupes dans un contexte économique de survie.

Le contexte de l'urbanisation

9 Les statistiques sont concordantes pour estimer qu'en l'an 2000, la moitié de la population africaine vivra dans les villes. L'Afrique Noire en fournit une illustration car elle est actuellement au monde la région où le taux d'urbanisation croît le plus rapidement, passant de 12 à $30 \%$ entre 1950 et 1980 . Cette concentration urbaine s'accompagne d'une croissance particulièrement rapide de la population de ce continent en dépit de son niveau de développement singulièrement faible et inquiétant.

10 Avec un taux annuel d'accroissement avoisinant $3 \%$, la population de l'Afrique subsaharienne doublera en l'espace de 23 ans, devenant ainsi la région du monde où la croissance est la plus rapide. La population urbaine a été multipliée par cinq dans le même temps. Deux phénomènes convergent pour expliquer une telle particularité : d'une part, une croissance naturelle élevée et, d'autre part, d'importants flux migratoires provenant du milieu rural en direction des villes, notamment les capitales.

11 Selon l'enquête IFAN/ORSTOM, paradoxalement le continent est peu urbanisé comparativement à l'Europe ou à l'Amérique Latine pour ne citer que ces exemples. Les villes africaines restent de dimension modeste. La moitié de la population urbaine vit encore dans des villes de moins d'un million d'habitants (80 \% en 1970, 69\% en 1985). Mais la proportion de la population urbaine demeurant dans des villes ayant plus d'un million d'habitants est passé de $19 \%$ en 1970 à $31 \%$ en 1985, et le nombre de villes millionnaires s'accroît (Antoine et al. 1992). Le fait urbain est incontestablement une nouvelle donne dans ce continent où la plupart des pays sont sous ajustement structurel.

12 Le Sénégal n'échappe pas à cette dynamique urbaine devenue irrésistible du fait de la crise profonde de son agriculture, pilier de l'économie nationale. Dakar n'est pas non plus à l'abri de la macrocéphalie caractéristique du modèle urbain africain où la capitale se développe de façon vertigineuse en écrasant les villes secondaires. Selon le dernier recensement de la population de 1988, Dakar et son "double", Pikine, comptaient 1.310.000 habitants. L'agglomération dakaroise accueille ainsi $19 \%$ de la population totale du pays et près de $54 \%$ de la population urbaine estimée à $39 \%$ de la population totale. L'accroissement de la population urbaine est rapide car il est annuellement de 3,8\% entre 1976 et 1988, alors que pour la même période l'accroissement annuel de la population est de l'ordre de $2,7 \%$.

13 L'essentiel des installations de l'économie moderne est implanté dans la capitale. Cependant ce pôle attractif est incapable d'offrir des emplois dans le secteur structuré à 
une bonne part des résidents ; faute de mieux, plus de la moitié des actifs exercent dans le secteur informel et le taux de chômage est estimé à $22 \%$ de la population active (Boquier \& Fall, 1992).

14 Ainsi que le notent Antoine et Savané (1990) l'urbanisation au Sénégal en particulier, et en Afrique indépendante en général, n'est ni le corollaire ni le moteur d'un quelconque développement économique; contrairement à ce qu'on a observé dans le monde industrialisé, elle n'a pas été entraînée par une mécanisation de l'agriculture ayant dégagé une surpopulation relative. Elle résulte plutôt de la dégradation rapide des conditions de vie dans les villages, et donc fondamentalement de la crise de l'agriculture. L'urbanisation apparaît donc comme un important défi ; la vile n'est donc pas dans ce cas un cadre alternatif de développement, elle est bien plutôt un espace de survie des individus et des ménages qu'ils constituent ou les entretiennent à distance.

Les relations à distance milieu urbain/milieu rural

15 Les processus migratoires s'enracinent souvent dans des traditions de mobilité spatiale qui remontent parfois à quelques générations. Le migrant est membre d'une communauté de parenté, de famille et de lignage souvent étendue, et laisse des membres de sa communauté dans le village pour en trouver d'autres anciennement installés en ville. Il maintient, par conséquent, des liens plus ou moins forts avec son milieu d'origine. D'autre part, les associations de ressortissants, qui réunissent des originaires d'une même région ou d'un même village, sont actives en ville. Ces associations jouent un rôle clé pour maintenir des liens privilégiés avec le milieu d'origine, ou éviter l'assimilation totale avec le milieu d'accueil, elles renforcent parfois le contrôle social comme dans le cas des jeunes bonnes Diola (Diop, 1989).

Il existe un flux important d'échanges et d'offres de vivres d'origine vers le milieu d'accueil. Des flux monétaires de la ville vers les milieux d'émigration sont aussi remarquables. De même, les envois d'argent sont volontaires. Néanmoins, l'effet du style de vie et des traditions communautaires encore vivaces en Afrique nous amène à relativiser cette autonomie de décision d'envoi monétaire par les migrants et quelques citadins à la faveur de leur communauté d'origine. En Afrique, les gens attendent énormément de leurs enfants. Peut-on parler réellement de solidarité si on sait que la norme sociale est fondée sur la primauté du groupe qui fonctionne dès lors comme une instance qui légifère? Les efforts pour rester soudé au groupe de base peuvent a contrario être perçus comme une reproduction de l'ordre social, un conformisme aux valeurs dominantes.

17 Les visites des ruraux et des migrants au milieu d'origine (villages, villes secondaires, pays...) sont des indicateurs du dynamisme des relations ville/village. Ces visites s'effectuent selon des circonstances variables. Dans notre enquête, près de $90 \%$ des migrants et $80 \%$ des migrantes entretiennent des relations avec la localité d'origine; mais le poids relativement important de ceux qui déclarent ne plus être allés dans la localité d'origine (16,5\% chez les migrants de la génération $25-34$ ans et $22,5 \%$ chez les jeunes migrantes) conduit à s'interroger sur les types de migration. Il n'est pas exclu que certains migrants aient rompu avec leur localité d'origine. La génération la plus jeune est plus portée à effectuer ces visites aussi bien pour les hommes que pour les femmes. Chez ces dernières, les cérémonies familiales l'emportent sur les fêtes religieuses : respectivement $61,2 \%$ et $38,5 \%$ pour les moins de 35 ans. Des transferts et échanges d'argent s'effectuent au cours de ces cérémonies L'émiettement des groupes structuraux villageois en milieu urbain n'aliène pas les liens socio-culturels entre 
ruraux et résidents dakarois. La migration temporaire en ville se maintient même si elle est mineure, du moins c'est ce que semblent indiquer des retours au village d'origine pour les travaux saisonniers agricoles et raisons assimilées. Là également les jeunes migrants sont plus concernés $(10,7 \%)$, par contre les migrantes retournent peu à peu à la localité d'origine pour les travaux saisonniers agricoles. Peu de migrants vont en congé dans leur localité d'origine, également peu de personnes effectuent des visites régulières (tableaux 1 et 2 ).

Une autre piste à explorer concerne les envois de fonds pour aider d'autres membres de la famille qui ne résident pas dans le ménage. Nous avons regroupé par génération, sexe et statut de migrant ou non, la proportion de personnes qui aident régulièrement une autre personne (la question exacte était: "Avez-vous des personnes à charge ou que vous assistez et qui ne logent pas avec vous ?") (tableaux 3 et 4 ).

Les dakarois naturellement soutiennent leurs noyaux conjugaux (le conjoint et les enfants) localisés dans la même ville tandis que les migrants envoient des dons aux noyaux conjugaux dans leur lieu d'origine. Les natifs de Dakar de même que les migrants de la génération 35-44 ans restent les plus concernés par les dons destinés au noyau conjugal. L'ensemble des dons intéressant le noyau conjugal consenti par cette génération est de 15,6\% pour les natifs de Dakar, et de 20,6\% pour les migrants. Pour toutes les générations, la contribution des migrants semble plus importante que celle des dakarois, ce qui s'explique par le fait qu'une part des migrants ont leur épouse à l'intérieur du pays.

20 Les jeunes dakarois entre 25 et 34 ans soutiennent leur père ou mère dans des proportions moins importantes (10,3\%) que ceux entre 35 et 44 ans (23\%). Lorsque leurs propres parents vivent dans la même ville qu'eux, ils sont également mieux soutenus par ces derniers ( $11,9 \%$ contre $3,6 \%$ chez les plus jeunes). Les migrants qui ont 44 ans ou moins envoient des dons plus importants à leurs propres parents et personnes assimilées dans la localité d'origine (43,8\%). Le soutien destiné exclusivement au père ou la mère vivant dans la localité d'origine est sensiblement le même pour les deux générations les plus jeunes (23,1\% pour les moins de 35 ans et $25 \%$ pour les moins de 45 ans). Les dons destinés au père ou la mère et à leurs co-résidents sont moins importants que les premiers (10,9\% pour les moins de 35 ans et $9,2 \%$ pour les moins de 45 ans) mais ils sont l'expression des liens multiples que les migrants continuent à entretenir à distance. Les migrants soutiennent néanmoins leurs propres parents installés à Dakar. Ce soutien est plus important chez les femmes (5,6\% pour les moins de 35 ans et 2,7\% pour les moins de 45 ans) que chez les hommes (2,3\% pour les moins de 35 ans et 2,6\% pour les moins de 45 ans) (tableaux 5, 6, 7 et 8).

21 L'effet de génération observé chez les hommes s'inverse pour les femmes aussi bien natives de Dakar que migrantes. Ainsi on peut relever que 18,5\% des dakaroises âgées de 25 à 34 ans soutiennent leur père ou mère et associés tandis que $10 \%$ seulement de celles âgées de 35-44 ans font des dons aux mêmes personnes. Les jeunes migrantes contribuent dans leur localité d'origine pour 31,5\% alors que celles plus âgées envoient au père ou mère et associés un peu moins (26,6\%). Parmi les destinataires, c'est évidemment le père ou la mère qui reçoivent le plus de soutien.

Les migrants les plus âgés sont plus préoccupés par l'envoi de don à la parenté en général. De tels dons sont plus importants pour ceux restés dans la localité d'origine. Cependant la solidarité entre migrants de la même parenté demeure effective et 
s'exprime entre autres par la participation financière aux associations qui sont surtout fonctionnelles à Dakar (tableaux 9 et 10).

En règle générale, les hommes de la génération 25-34 ans nés à Dakar, donnent peu, alors que ceux de la génération 45-59 ans, qui ont en général un ménage de taille déjà élevée, aident en plus d'autres membres de la parenté. Il y a là des éléments pour se demander avec Antoine (1992) si "les générations de l'indépendance (les 45-59 ans) ne sont pas une génération charnière qui supportent le plus le poids de la crise. Les jeunes vivent la crise, les aînés la supportent" (tableaux 11 et 12). La précarité des conditions de vie dans les localités d'origine des personnes enquêtées explique le peu de soutien qu'elles reçoivent. Le soutien du père ou de la mère, des frères ou des sœurs est important pour les jeunes dakarois (6,6\%). L'appui des collatéraux en faveur des migrants est effectif d'une génération à l'autre, néanmoins l'intérêt manifesté par les plus jeunes est notable (9,9\%). Les personnes âgées entre 45 et 59 ans particulièrement les dakarois sont soutenus par leur noyau conjugal $(6,3 \%)$. Cet état de fait peut être expliqué par l'implication des femmes polygames dans la gestion de la survie de leur ménage respectif. Les relations interpersonnelles informelles en marge des groupes d'origine semblent être d'un bon concours pour les dakarois comme pour les migrants ( $6,3 \%$ pour les premiers et $6,5 \%$ pour les seconds). Les soutiens reçus d'autres personnes sans lien de parenté ou d'alliance sont permanents pour toutes les générations, même si ils semblent moins établis pour la génération intermédiaire 35-44 ans. Les jeunes dakaroises ont la faveur de tels soutiens (16\%). On relève également des soutiens reçus des associations au profit des migrants et des migrantes.

Le recours aux réseaux pour l'accès au logement

Les institutions chargées des services pour l'accès au logement en ville sont peu structurées. Les relations interpersonnelles sont donc mises à contribution afin de satisfaire ce besoin. Le recours à un intermédiaire pour l'accès au logement est bien vécu et accepté. Aider une personne à trouver un logement est un fait naturel, banalisé. Toutefois le recours à un intermédiaire semble moins fréquent que nous le pensions lors de l'élaboration de l'étude. D'après les résultats de l'enquête environ 50 à $60 \%$ des migrants arrivés à l'âge de 20 ans n'ont pas eu recours à un intermédiaire pour trouver un logement. Cette proportion est plus faible pour la génération la plus jeune (tableau 13).

27 Le recours à la parenté prend de plus en plus d'importance. Alors que les générations précédentes n'y avait recours que dans $20 \%$ des cas, ce recours est plus fréquent pour les individus de la génération 25-34 ans (32,7\%).

28 Les migrants s'appuient parfois sur les amis dont le rôle n'est pas négligeable pour l'accès au premier logement. En effet $12,17 \%$ de la génération de 45 à 54 ans s'est appuyée sur des amis afin d'accéder au premier logement, tandis que 10,93\% de la génération suivante (35 à 44 ans) agit ainsi. Le recours est moins fréquent pour la génération la plus jeune $(2,8 \%)$. Ces intermédiaires résident en majorité dans le même voisinage que les migrants soutenus, et ce, quelle que soit la génération (entre 86 et $89 \%$ des cas) (tableau 14).

Lors de l'accueil des migrants dans l'agglomération de Dakar, les réseaux parentaux en ville jouent un rôle essentiel. Parmi les hommes hébergés ou vivants en co-location, 41 $\%$ vivent avec leur père (ou mère) ou un ascendant. Cette proportion est nettement plus 
importante que pour les générations précédentes. Le recours aux collatéraux est également fréquent. Par contre on vit plus rarement chez une tierce personne n'appartenant pas à la famille (5,33\% des cas pour la génération $25-34$ ans, et $16,87 \%$ pour la génération 45-54 ans). En règle générale, on peut retenir que "une famille de Dakar sur deux (et plus particulièrement les classes moyennes) accueille des migrants, à l'exception des dakarois de souche qui sont moins sollicités" (Antoine, 1991).

Recours aux réseaux pour le premier emploi

Le premier emploi constitue souvent un apprentissage de la capacité des travailleurs indépendants à assumer un nouveau statut professionnel. L'appel à des personnes-ressources n'intervient qu'une fois que l'individu a fait ses propres armes. Même si la volonté de réussir est réelle, on est peu tenté de faire partager davantage de risques à ses proches. Le recours à un intermédiaire est de plus en plus pratiqué d'une génération à l'autre tant pour les personnes non natives de Dakar que pour les dakarois. La sollicitation adressée aux personnes "sans lien de parenté" est dérisoire. Cela est attribuable à la faiblesse des réseaux extra-familiaux et professionnels lors de la première période d'installation des indépendants, aussi bien pour les migrants que pour les natifs de Dakar. Ceux qui ont trouvé un emploi peu après leur arrivée à Dakar (tableau 15) consultent de plus en plus un parent parmi les ascendants et les collatéraux (11,67\% pour la génération des $45-59$ ans et $34 \%$ pour la dernière génération 25-34 ans), et relativement peu aux autres parents $(1,04 \%)$ ou aux individus avec lesquels ils n'ont pas de lien de parenté ou d'alliance $(3,13 \%)$. Le recours à la parenté était moins fréquent pour les générations précédentes, même si le rôle du père reste prépondérant. Ceux qui ont connu une période d'attente après leur arrivée à Dakar avant d'accéder à un premier emploi (tableau 16) font aussi, d'une génération à l'autre de plus en plus appel à un intermédiaire qui est avant tout un ascendant. On constate dans le cas des indépendants nés à Dakar, que la personne prêtant ses ressources matérielles ou relationnelles vit avec celui qu'elle soutient et appartient à son réseau d'origine.

31 Pour les indépendants nés hors de Dakar, la génération la plus jeune a davantage recours que les précédentes à des personnes résidant hors du logement. Les indépendants qui ont suivi tout ou partie de leur formation à Dakar (tableaux 16 et 17), alors qu'ils n'avaient eu recours à pratiquement personne dans les générations âgées de 45-59 ans, plus de 50\% des migrants et non migrants n'ont eu recours à personne. Parmi les intermédiaires, les non migrants ont de plus en plus recours au père et aux oncles et autres parents.

Nos entretiens montrent que l'engagement au travail indépendant, quoique longuement mûri par le concerné, correspond à une décision en apparence spontanée, souvent brutalement prise à la suite d'un conflit avec le patron. Une telle décision reflète bien plus une volonté de démontrer la maîtrise de son métier, et que l'on ne saurait exprimer totalement sous l'ombre d'un patron, l'apprentissage n'ayant que trop duré. C'est aussi, dans certaines circonstances, une expression d'affranchissement vis-à-vis d'un modèle traditionnel de rapports de dépendance professionnelle.

Le salariat auprès d'un parent n'est pourtant pas une alternative ou un substitut en vigueur. En effet, on est rarement salarié au service d'un parent quelle que soit la génération (moins de $4 \%$ des cas pour les migrants juste après leur arrivée à Dakar). S'agissant cependant des hommes de moins de 35 ans, il semblerait que la tendance à être salarié chez un parent émerge ( $9 \%$ des cas) après une période de chômage ou 
d'étude ; alors que le cas était très rare pour les générations précédentes. Par contre, quelque soit leur statut migratoire, aucune femme salariée du même âge n'avait travaillé chez un parent pour un premier emploi. Mais il va de soi que cet élan demeure à vérifier. Généralement le recours à un parent ne prend pas la forme d'une demande d'emploi. En revanche les réseaux parentaux sont davantage mobilisés par les jeunes générations en particulier au moment de l'arrivée à Dakar (tableaux 18 et 19). Le recours à un intermédiaire pour l'obtention d'un emploi salarié concerne entre un quart et une moitié des individus selon la génération et le statut migratoire: on remarquera une tendance chez les enquêtés formés à Dakar à s'appuyer de plus en plus sur un tiers pour obtenir leur premier emploi salarié, alors que pour les migrants venus chercher du travail, ce serait plutôt à la fois les générations âgées et les plus jeunes.

Chez les femmes, la médiation au service de l'accès à l'emploi salarié est en faveur des natives de Dakar, à raison de $45,71 \%$ contre $33,33 \%$ pour les migrantes ayant eu leur emploi immédiatement et seulement $26,83 \%$ pour celles qui ont connu une période d'attente. Alors que les migrantes des générations plus anciennes avaient peu recours à un intermédiaire, ce recours s'accroît et est du même ordre que pour les dakaroises qui, quelle que soit la génération, recourt dans la moitié des cas à un intermédiaire. De manière générale, l'accès à un emploi salarié par le recours à un intermédiaire est fréquent mais pas majoritaire.

35 À l'échelle des lieux de travail, l'étroitesse du marché de l'emploi va de paire avec le cloisonnement de l'information sur les éventuelles ouvertures de postes et admissions comme apprenti avec ou sans promesse de recrutement futur. Les personnes les plus proches intervenues pour la recherche d'un emploi salarié travaillent plus fréquemment dans la même entreprise de ceux qu'ils ont soutenu, et ce, indépendamment du statut migratoire avec toutefois des tendances inverses : c'est de moins en moins le cas pour ceux nés hors de Dakar et de plus en plus le cas pour les dakarois. Cela reflète les procédés de cooptation intervenant à partir du lieu de travail devenu une zone de pouvoir dont le contrôle n'est pas du seul ressort des décideurs. On peut faire l'hypothèse d'une proximité sociale reliant les intermédiaires à leurs protégés, ce qui indique le maintien de leurs liens d'origine (tableaux 23 et 24).

L'examen du lieu de résidence des intermédiaires conduit cependant à tempérer cette assertion. En effet, moins d'un tiers des intermédiaires partagent le même logement que leurs protégés exceptés les migrants formés hors Dakar/Pikine et nés après 1945 qui semblent davantage avoir recours à un tiers habitant le même logement. Le recours à des personnes travaillant dans les mêmes entreprises est néanmoins une pratique plus fréquente chez les générations migrantes les plus âgées. Le phénomène inverse est observé chez les natifs de Dakar où les moins de 35 ans trouvent plus d'emploi que les autres au sein des entreprises où travaillent leurs protecteurs. Chez les femmes, 27,27\% des migrantes accédant à un emploi salarié dès l'arrivée à Dakar sollicitent un intermédiaire sur le lieu de travail. L'appartenance au même lieu de travail et hors entreprise des natives de Dakar est semblable (50\%). Par contre, dans le cas des femmes nées à l'extérieur de Dakar et ayant attendu leur premier emploi, la catégorie "même entreprise" l'emporte légèrement $(55,56 \%)$. Les migrantes ayant trouvé du travail dès leur arrivée à Dakar ont plus souvent recours à une femme (72,73\%). On peut expliquer cet état de fait par l'exercice du travail domestique. Inversement, les femmes nées à Dakar ainsi que les migrantes ayant connu une période d'attente mobilisent deux fois plus d'hommes. Les hommes quant à eux ne sollicitent guère l'intervention féminine. 
En règle générale, on peut conclure que les femmes font plus appel aux femmes que les hommes, mais elles mettent majoritairement à contribution les hommes afin de satisfaire leurs besoins d'accession à l'emploi salarié (tableaux 25, 26, 27 et 28).

Les grandes lignes du recours à un intermédiaire pour l'accès à l'emploi se présentent ainsi qu'il suit : s'agissant des hommes salariés, ils recourent dans une large majorité à d'autres hommes. En général, il n'y a pas de recours systématique à une personne dans la parenté. En dépit des critères de recrutement supposés basés sur la compétence dans le salariat, on relève l'effectivité d'un recours à un intermédiaire même dans le secteur public. Dans l'ensemble, pour le salariat, on observe un report du recours vers les collatéraux dans le cas de ressources limitées auprès des ascendants : c'est le cas des nouveaux arrivants à Dakar et les jeunes générations.

Les hommes travaillant comme indépendants sollicitent beaucoup moins d'intermédiaire que les salariés. Ils comptent plus sur eux-mêmes. Cependant, à la différence des salariés, et des indépendants âgés ou migrants, les jeunes dakarois (génération née entre 1955 et 1964) mobilisent fréquemment leur père pour accéder à l'emploi. Il semblerait donc que la transmission de patrimoine de père en fils commence à montrer ses effets chez les travailleurs indépendants, plus particulièrement dans le domaine de la production et des services.

39 Comparativement aux hommes qui, rappelons-le, ne sollicitent presque pas de femme pour obtenir un emploi, les femmes ont recours de manière plus équilibrée à des hommes et à des femmes. A l'exception des migrantes exerçant comme indépendantes dans la production et les services, les femmes, lorsqu'elles ont recours à un tiers, sollicitent quasi systématiquement leur époux pour travailler à leur compte propre. Mais comme pour les hommes, on observe chez les femmes plus de cas de non recours à un intermédiaire pour l'accession à l'emploi.

Critique du continuum rural-urbain

40 Les récits de vie collectés auprès des migrants à Dakar et Pikine confirment que les associations de ressortissants ou d'originaires de villages ou de communautés rurales ainsi que les regroupements familiaux, lignagers et ethniques en ville sont d'importants relais pour l'accueil et l'insertion des migrants. Des personnes reliées par une origine commune et pouvant appartenir à des catégories sociales différentes s'y côtoient et partagent leurs préoccupations. Mais comme toute communauté d'origine, de telles associations ont une logique normative. Elles constituent un système lourd de fonctionnement et conservateur du point de vue des valeurs reproduites. Les besoins quotidiens des membres sont difficilement pris en charge. Il arrive également que les valeurs socio-culturelles entretenues se heurtent aux velléités modernistes que les besoins d'adaptation ou de créativité urbaines des membres mettent en relief. Dès lors, c'est en marge du système que se développent potentiellement des réseaux et segments de réseaux. Or O'Deye (1985) présente ces associations d'originaires et de ressortissants comme un moyen d'intégration au sein du lignage tout en servant de réceptacle des velléités opposées au sein du groupe des migrants organisés dans cette entité. Ainsi "la ville reconstruit les associations" (O'Deye : 1985, 37). Au contraire de cette approche pour le moins ruraliste, la ville, Dakar en particulier, n'est pas à considérer comme un lieu privilégié de prolongements des relations traditionnelles villageoises. Nous avons plutôt observé l'émergence de nouvelles formes de relations résultant de la dynamique de sociabilités urbaines. L'acteur urbain est un faiseur de réseaux. La restructuration permanente des réseaux de sociabilité semble être une caractéristique essentielle de 
l'urbanisation dakaroise. En effet avec l'aggravation de la crise économique et les contraintes de la vie urbaine, les acteurs sociaux développent des stratégies visant l'élargissement de leur espace de sociabilité. L'émergence de nouvelles relations en ville est désormais un indicateur de la dynamique d'insertion urbaine des migrants à Dakar et Pikine. Les réseaux sociaux spécifiquement urbains contribuent à affaiblir les liens ville/ village.

Maintien des réseaux ruraux et importance des réseaux urbains

41 Les travaux de recherche font de plus en plus de place aux rôles privilégiés des réseaux sociaux, comme phénomène construit d'accueil et d'insertion urbaine des migrants. Leur fonction "d'amortisseur" de la crise économique et sociale généralisée se heurte à la problématique de leur permanence et de leur renouvellement. L'effet de la dynamique de groupe aidant, les réseaux d'origine (structures familiales, communautés ethniques, villageoises...) occupent une position centrale au moment de la préparation des candidats à la migration et aussi lors des premières étapes de l'installation à la ville avant de se laisser relayer, dans les étapes futures, par des réseaux plus spécifiquement urbains, tout en étant l'une des formes dominantes du processus d'insertion en ville, est insuffisamment mentionné et analysé dans les travaux récents sur les migrations en Afrique.

Comme on le sait, des réseaux sociaux, familiaux, culturels, religieux se sont ainsi constitués et consolidés sur la base de l'esprit de solidarité et de la survie des communautés impliquées. Ces réseaux sont des instruments efficaces d'accueil, d'entraide, de recherche d'emploi et de logement pour le migrant. Tout en s'organisant sur les rapports traditionnels de solidarité mais aussi de hiérarchie et d'inégalité entre les membres, les réseaux se "modernisent" en quelque sorte, en intégrant d'autres types de solidarité et de liens créés par de nouvelles appartenances: relations informelles, de voisinage, politiques, religieuses, sportives, syndicales et professionnelles.

La permanence des réseaux d'origine

Ce contexte de la migration rurale-urbaine met en relief le rôle des réseaux d'origine, tout au moins dans le processus d'accueil des migrants à Dakar. De même, les réseaux parentaux, ethniques, et d'autres types de réseaux bâtis autour de l'appartenance à une même aire régionale et socio-culturelle (région, département, communauté rurale, village...) interviennent plus ou moins fortement pour l'accès du nouveau migrant au premier logement et au premier emploi ou occupation professionnelle. Evidemment, ils sont plus accessibles que les réseaux intégrés ou créés avec un temps de présence plus important en ville. Mais les réseaux d'origine ne sont pas non plus des relais sociaux existant d'avance et qu'il suffit d'intégrer en cas de besoin. Ils sont naturellement des phénomènes élaborés, construits différemment en fonction des ressources propres à chaque espace relationnel impliquant les acteurs concernés ou des communautés structurées ou informelles. On peut néanmoins relever la permanence des réseaux d'origine dans ce processus migratoire. C'est ce qui a fait dire à Antoine et Savané (1990: 56) : "la migration entretient la migration". Selon ces auteurs la migration résulte des membres d'une famille. Ainsi les migrants s'appuient sur des réseaux relationnels en vue de leur insertion en ville. Le pont est donc établi entre le milieu rural et la ville. Les migrants occupant des postes à responsabilité en ville réinvestissent parfois, économiquement et socialement, dans leur région d'origine : la participation à la construction d'écoles, de dispensaires, de nouvelles maisons, aux 
achats d'équipements agricoles, et également participation à la vie politique et sociale : le maintien de relations avec le milieu d'origine entraîne d'importants flux financiers entre la ville et la campagne.

Les pôles de la famille se multiplient. Des circuits d'accueil et d'insertion s'instaurent dans la ville. Les transferts de fonds, de vivres, de médicaments etc. sont effectués. C'est la famille au sens élargi qui se déploie. Au Sénégal, des femmes appartenant à des ménages polygames prennent leur tour en ville ou au village sous forme de rotation pendant que le mari fait son expérience urbaine. On peut donc noter l'existence d'une composante urbaine des stratégies rurales et inversement.

L'émergence de nouvelles formes de relations à Dakar

Les réseaux ne sont pas référés à des structures organisées dont le mode de fonctionnement est explicite. A force de prospecter plus largement, on découvre la richesse des relations informelles révélant d'autres mécanismes de mise en place de réseaux. On peut repérer dans la ville plusieurs exemples de regroupements informels : des voisins s'asseyant devant l'atelier d'un tailleur presque tous les jours depuis plusieurs années ; une "grande place" où des personnes jouent à la belote ou aux dames depuis une trentaine d'années; des courtiers qui monnayent leur expertise de l'intermédiation pour la vente et la location de logements, des cadres intellectuels jouant aux boules le week-end. Afin de montrer le champ assez grand de noyaux relationnels pouvant être dynamisés pour générer des réseaux sociaux, quelques cas de figure sont brièvement évoqués dans ce qui suit.

Du fait du rétrécissement progressif du marché de l'emploi salarié occasionné entre autres par la politique d'ajustement structurel, le secteur de l'économie informelle se présente de plus en plus comme la porte d'entrée privilégiée des migrants dans le tissu économique dakarois. La sous-qualification professionnelle des nouveaux migrants en est une explication. L'économie informelle est relativement souple pour autoriser l'accueil des nouveaux migrants préoccupés d'avoir un métier pour s'insérer durablement en ville. Pour se former, les migrants s'appuient davantage sur des réseaux familiaux.

47 Mais à mesure que se déroule l'apprentissage, le migrant s'implique dans des réseaux que génèrent ses rapports de prestation de services pour une partie de la clientèle de l'atelier où il travaille. Son installation future comme indépendant dépendra largement de sa capacité à fidéliser sa clientèle acquise à la défaveur de son patron et surtout des possibilités de profiter de ses réseaux de relations qu'il aura suscités tout au long de son apprentissage.

Nous avons observé que les migrants ayant réussi dans l'économie informelle s'impliquent activement dans d'autres réseaux confrériques, regroupements d'opérateurs économiques, et même au sein des réseaux de commerçants. Pour réussir dans l'informel, il apparaît nécessaire de jouer des rôles importants dans des réseaux d'ascension sociale. En effet, les réseaux confrériques se sont beaucoup développés à partir de l'économie informelle. Cette forte interdépendance s'explique par le caractère socio-économique des confréries religieuses. A ce titre, leur fonction de groupe de pression socio-politique attire une clientèle de plus en plus nombreuse, en particulier tous ceux qui sont appelés à se repositionner et à conquérir de nouveaux statuts.

49 Le principe des vases communicants est applicable entre l'économie informelle, les migrations et les réseaux confrériques. On constate des convergences quasi-naturelles qui favorisent le passage de l'un à l'autre. Les réseaux fondés sur les religions peuvent 
être considérés comme des réseaux d'origine car l'adhésion à une religion participe au processus de socialisation. On ne choisit pas sa religion, on l'hérite de ses parents. La religion est un élément culturel d'origine. En revanche, l'appartenance à des associations religieuses et de plus en plus à des confréries religieuses peut procéder des deux registres d'origine et optionnel. Le passage des réseaux villageois aux réseaux optionnels est ici à mettre au compte de la plasticité des réseaux confrériques et du caractère populaire de l'économie informelle à Dakar et Pikine.

Dans le secteur structuré, les relations professionnelles sont pour la plupart activées, générant ainsi des réseaux sociaux typiquement urbains. Les syndicats constituent un espace de sociabilité favorisant l'émergence de réseaux à l'échelle et hors de l'entreprise. A partir d'une désignation comme délégué du personnel, un quelconque travailleur accède à des informations souterraines lui conférant une parcelle de pouvoir au sein de l'entreprise. Ce pouvoir de médiateur est souvent le moyen d'accéder à des faveurs lui permettant de placer parents ou amis à un nouveau poste et d'avoir ses protégés et ses protecteurs au sein de l'entreprise, aussi bien au niveau de la direction que parmi les agents. Le style militant des activités d'entreprises différentes sont autant de facteurs à la fois objectifs et subjectifs facilitant la création d'espaces de sociabilité et de réseaux sociaux en milieu urbain. La culture ouvrière est une composante de la culture typiquement urbaine.

51 L'intensité de la vie professionnelle dans l'entreprise et la "proximité interindividuelle" favorisent les réseaux d'amitié. En effet, au sein de l'entreprise, les travailleurs vivent souvent en commun des épreuves de tous genres qui resserrent leurs liens. La durée d'emploi dans l'entreprise fait que les lieux de travail permettent le développement de relations continues et l'approfondissement de la connaissance interindividuelle. Ces relations d'amitié se prolongent hors de l'entreprise et fraient la voie à des réseaux sociaux denses et d'une vitalité insoupçonnée.

Les associations religieuses ou "daayira" au sein des entreprises sont également un moyen courant d'activer les relations interprofessionnelles. Les réseaux sociaux qui se mettent en place à la faveur de la même appartenance confrérique donnent de l'intérêt aux relations par petits groupes au sein de l'entreprise. Ils sont les moyens de "socialiser" la vie de l'entreprise et réciproquement de prolonger les relations professionnelles privilégiées hors de l'entreprise (Diop, 1982; Ndiaye, 1989). Tout comme les rapports de travail, les relations contingentes de voisinage peuvent subir des transformations et développements générant des réseaux promotionnels. Il est établi que le voisinage fait partie intégrante de leur identité sociale. On peut également considérer que les migrants, notamment ceux bénéficiant d'une longue expérience résidentielle à Dakar, s'accommodent de cette référence identitaire (Fall, 1991b). Les réseaux de voisinage, les réseaux informels et les réseaux religieux confrériques occupent de nouveaux espaces créés en ville. Ils constituent, par leur grande accessibilité, des moyens privilégiés d'insertion sociale dans le contexte urbain dakarois. Une autre caractéristique non moins importante de ces réseaux est la meilleure intégration de l'anonymat et la consolidation d'un brassage à la portée du public.

53 Conclusion

54 Le passage des réseaux d'origine aux réseaux urbains ne relève pas d'un procédé balistique. Il correspond plutôt à un processus heurté et discontinu. L'originalité des réseaux sociaux réside dans le fait qu'ils procèdent tout à la fois de stratégies des 
acteurs du dedans comme du dehors des institutions sociales en vue de la satisfaction de leurs besoins. Ils se constituent et se fortifient en mettant à contribution, au cas où c'est nécessaire, les ressources propres ou potentielles des institutions, ou inversement, à les contourner en se développant sur leurs marges, comme pour défier la rigidité des systèmes et laisser se profiler les changements en termes de réadaptation ou de rupture. Les réseaux s'estompent au fur et à mesure que les besoins des acteurs sociaux qui les animent sont satisfaits ou nécessitent d'autres relais sociaux. Ils peuvent donc être déviés de leur raison sociale fondatrice.

La stratégie de contrôler des "zones de pouvoir" au sein ou à l'échelle de plusieurs institutions rend nécessaires les réseaux sociaux. Les réseaux traversent les institutions et transgressent leurs limites. Les réseaux n'ont pas pour rôle de se substituer aux institutions ; ils tentent plutôt de les dépasser et de les vivifier en les dynamisant.

Le migrant n'a pas besoin de s'opposer aux institutions sociales d'origine pour réussir son insertion urbaine. Les réseaux urbains s'édifient sur l'espace de sociabilité comme moyen de conquérir d'autres zones de possibilités favorables à l'insertion des migrants en ville. Le passage des uns aux autres s'opère concomitamment à l'élargissement et à la complexification de l'espace de sociabilité du migrant en ville. Réseaux villageois et réseaux urbains conservent leur logique informelle et implicite. Mais les réseaux d'origine ne s'apparentent pas totalement aux réseaux villageois qui n'en sont qu'une composante. La permanence des réseaux d'origine n'a d'égale que la vitalité des réseaux sociaux urbains qui constituent désormais un centre d'intérêt privilégié pour l'analyse de la dynamique d'insertion des migrants à Dakar et Pikine. Il est permis de dire avec Pontié et Lericollais (1991) que "les réseaux de solidarité structuraux ne sont pas marginalisés par la migration". De même, il demeure établi que le développement fulgurant de nouveaux réseaux sociaux permettant $d$ "'amortir" la crise - les relations à distance étant une des modalités de cette solidarité - va de paire avec l'émiettement des groupes structuraux.

Tableau 1 : Type de visite des migrants dans leur localité d'origine par génération

\begin{tabular}{|c|c|c|c|}
\hline & \multicolumn{3}{|c|}{ Générations } \\
\hline Raisons de la visite & $25-34$ ans & $35-44$ ans & $45-59$ ans \\
\hline Travaux saisonniers et raisons assimilées & $\begin{array}{l}24 \\
10,7 \%\end{array}$ & $\begin{array}{l}12 \\
5,3 \%\end{array}$ & $\begin{array}{l}14 \\
5,4 \%\end{array}$ \\
\hline Travaux saisonniers seulement & $\begin{array}{l}2 \\
0,9 \%\end{array}$ & $\begin{array}{l}1 \\
0,4 \%\end{array}$ & $\begin{array}{l}1 \\
0,4 \%\end{array}$ \\
\hline Fêtes religieuses et cérémonies familiales & $\begin{array}{l}66 \\
29,4 \%\end{array}$ & $\begin{array}{l}60 \\
26,3 \%\end{array}$ & $\begin{array}{l}46 \\
17,7 \%\end{array}$ \\
\hline Congés et fêtes religieuses et cérémonies familiales & $\begin{array}{l}16 \\
7,1 \%\end{array}$ & $\begin{array}{l}11 \\
4,8 \%\end{array}$ & $\begin{array}{l}5 \\
1,9 \%\end{array}$ \\
\hline
\end{tabular}




\begin{tabular}{|c|c|c|c|}
\hline Congés et cérémonies familiales et divers & $\begin{array}{l}4 \\
1,8 \%\end{array}$ & $\begin{array}{l}18 \\
7,9 \%\end{array}$ & $\begin{array}{l}11 \\
4,2 \%\end{array}$ \\
\hline Congés et cérémonies familiales & $\begin{array}{l}5 \\
2,2 \%\end{array}$ & $\begin{array}{l}20 \\
8,8 \%\end{array}$ & $\begin{array}{l}17 \\
6,5 \%\end{array}$ \\
\hline Congés et visites périodiques & $\begin{array}{l}0 \\
0\end{array}$ & $\begin{array}{l}2 \\
0,9 \%\end{array}$ & $\begin{array}{l}1 \\
0,4 \%\end{array}$ \\
\hline Congés seulement & $\begin{array}{l}4 \\
1,8 \%\end{array}$ & $\begin{array}{l}5 \\
2,2 \%\end{array}$ & $\begin{array}{l}6 \\
2,3 \%\end{array}$ \\
\hline Cérémonies familiales et visites périodiques & $\begin{array}{l}2 \\
0,9 \%\end{array}$ & $\begin{array}{l}1 \\
0,4 \%\end{array}$ & $\begin{array}{l}9 \\
3,4 \%\end{array}$ \\
\hline Cérémonies familiales et autres & $\begin{array}{l}22 \\
9,8 \%\end{array}$ & $\begin{array}{l}24 \\
10,5 \%\end{array}$ & $\begin{array}{l}44 \\
16,9 \%\end{array}$ \\
\hline Cérémonies familiales seulement & $\begin{array}{l}22 \\
10,3 \%\end{array}$ & $\begin{array}{l}37 \\
16,2 \%\end{array}$ & $\begin{array}{l}55 \\
21,1 \%\end{array}$ \\
\hline Visites périodiques & $\begin{array}{l}1 \\
0,4 \%\end{array}$ & $\begin{array}{l}1 \\
0,4 \%\end{array}$ & $\begin{array}{l}2 \\
0,8 \%\end{array}$ \\
\hline Fêtes religieuses seulement & $\begin{array}{l}5 \\
2,2 \%\end{array}$ & $\begin{array}{l}1 \\
0,4 \%\end{array}$ & $\begin{array}{l}3 \\
1,1 \%\end{array}$ \\
\hline Autres raisons & $\begin{array}{l}12 \\
5,4 \%\end{array}$ & $\begin{array}{l}15 \\
6,6 \%\end{array}$ & $\begin{array}{l}10 \\
3,8 \%\end{array}$ \\
\hline Ne va jamais & $\begin{array}{l}35 \\
16,5 \%\end{array}$ & $\begin{array}{l}19 \\
8,3 \%\end{array}$ & $\begin{array}{l}35 \\
13,8 \%\end{array}$ \\
\hline & 220 & 228 & 260 \\
\hline Total & $100 \%$ & $100 \%$ & $100 \%$ \\
\hline
\end{tabular}

Tableau 2 : type de visite des migrantes dans leur localité d'origine par génération

\begin{tabular}{|l|l|l|l|}
\hline \multicolumn{2}{|l|}{} & \multicolumn{2}{|l|}{ Générations } \\
\hline \hline Raisons de la visite & $25-34$ ans & $35-44$ ans & $45-59$ ans \\
\hline \hline Travaux saisonniers et raisons assimilées & 0 & 3 & 1 \\
& 0 & $2,7 \%$ & $0,8 \%$ \\
\hline
\end{tabular}




\begin{tabular}{|c|c|c|c|}
\hline Fêtes religieuses et cérémonies familiales & $\begin{array}{l}13 \\
14,6 \%\end{array}$ & $\begin{array}{l}24 \\
12,4 \%\end{array}$ & $\begin{array}{l}13 \\
9,8 \%\end{array}$ \\
\hline Congés et fêtes religieuses et cérémonies familiales & $\begin{array}{l}2 \\
2,2 \%\end{array}$ & $\begin{array}{l}1 \\
0,9 \%\end{array}$ & $\begin{array}{l}0 \\
0\end{array}$ \\
\hline Congés et cérémonies familiales et divers & $\begin{array}{l}2 \\
2,2 \%\end{array}$ & $\begin{array}{l}0 \\
0\end{array}$ & $\begin{array}{l}2 \\
1,5 \%\end{array}$ \\
\hline Congés et cérémonies familiales & $\begin{array}{l}1 \\
1,1 \%\end{array}$ & $\begin{array}{l}3 \\
2,7 \%\end{array}$ & $\begin{array}{l}0 \\
0\end{array}$ \\
\hline Congés seulement & $\begin{array}{l}0 \\
0\end{array}$ & $\begin{array}{l}1 \\
0,9 \%\end{array}$ & $\begin{array}{l}1 \\
0,8 \%\end{array}$ \\
\hline Cérémonies familiales et visites périodiques & $\begin{array}{l}1 \\
1,1 \%\end{array}$ & $\begin{array}{l}1 \\
0,9 \%\end{array}$ & $\begin{array}{l}1 \\
0,8 \%\end{array}$ \\
\hline Cérémonies familiales et autres & $\begin{array}{l}16 \\
18,0 \%\end{array}$ & $\begin{array}{l}22 \\
19,5 \%\end{array}$ & $\begin{array}{l}34 \\
25,6 \%\end{array}$ \\
\hline Cérémonies familiales seulement & $\begin{array}{l}26 \\
29,2 \%\end{array}$ & $\begin{array}{l}42 \\
37,2 \%\end{array}$ & $\begin{array}{l}43 \\
32,3 \%\end{array}$ \\
\hline Visites périodiques & $\begin{array}{l}0 \\
0\end{array}$ & $\begin{array}{l}0 \\
0\end{array}$ & $\begin{array}{l}2 \\
1,5 \%\end{array}$ \\
\hline Fêtes religieuses seulement & $\begin{array}{l}2 \\
2,2 \%\end{array}$ & $\begin{array}{l}1 \\
0,9 \%\end{array}$ & $\begin{array}{l}0 \\
0\end{array}$ \\
\hline Autres raisons & $\begin{array}{l}5 \\
5,6 \%\end{array}$ & $\begin{array}{l}9 \\
8,0 \%\end{array}$ & $\begin{array}{l}9 \\
6,8 \%\end{array}$ \\
\hline Ne va jamais & $\begin{array}{l}20 \\
22,5 \%\end{array}$ & $\begin{array}{l}16 \\
14,2 \%\end{array}$ & $\begin{array}{l}28 \\
21,1 \%\end{array}$ \\
\hline & 88 & 113 & 132 \\
\hline Total & $100 \%$ & $100 \%$ & $100 \%$ \\
\hline
\end{tabular}

Tableau 3 : Don au noyau conjugal par génération. Hommes nés à Dakar 


\begin{tabular}{|c|c|c|c|c|c|c|c|c|}
\hline Générations & $\begin{array}{l}\text { Aucun } \\
\text { don } \\
0,00\end{array}$ & $\begin{array}{l}\text { Autre don } \\
\text { ailleurs } \\
1,00\end{array}$ & $\begin{array}{l}\text { Nc. } \\
\text { Autre } \\
\text { don } \\
2,00\end{array}$ & $\begin{array}{l}\text { Nc ail + } \\
\text { L. orig. } \\
3,00\end{array}$ & $\begin{array}{l}\text { Nc. } \\
4,00\end{array}$ & $\begin{array}{l}\text { Nc Dakar + } \\
\text { autre don } \\
6,00\end{array}$ & $\begin{array}{l}\text { Nc } \\
\text { Dakar } \\
\text { row } \\
7,00\end{array}$ & TOTAL \\
\hline $\begin{array}{l}1,00 \\
25-34 \text { ans }\end{array}$ & $\begin{array}{l}98 \\
59,0 \%\end{array}$ & $\begin{array}{l}52 \\
31,3 \%\end{array}$ & $\begin{array}{l}2 \\
1,2 \%\end{array}$ & & & $\begin{array}{l}7 \\
4,2 \%\end{array}$ & $\begin{array}{l}7 \\
4,2 \%\end{array}$ & 166 \\
\hline $\begin{array}{l}2,00 \\
35-44 \text { ans }\end{array}$ & $\begin{array}{l}30 \\
27,5 \%\end{array}$ & $\begin{array}{l}62 \\
56,9 \%\end{array}$ & $\begin{array}{l}4 \\
3,7 \%\end{array}$ & $\begin{array}{l}1 \\
0,9 \%\end{array}$ & $\begin{array}{l}2 \\
1,8 \\
\%\end{array}$ & $\begin{array}{l}8 \\
7,3 \%\end{array}$ & $\begin{array}{l}2 \\
1,8 \%\end{array}$ & 109 \\
\hline $\begin{array}{l}3,00 \\
45-59 \text { ans }\end{array}$ & $\begin{array}{l}18 \\
28,1 \%\end{array}$ & $\begin{array}{l}41 \\
61,1 \%\end{array}$ & & & & $\begin{array}{l}5 \\
7,8 \%\end{array}$ & & 64 \\
\hline
\end{tabular}

Tableau 4 : Don au noyau conjugal par génération. Migrant

\begin{tabular}{|c|c|c|c|c|c|c|c|c|c|}
\hline & Destin & aires & & & & & & & \\
\hline $\begin{array}{l}\text { Générat } \\
\text { ions }\end{array}$ & $\begin{array}{l}\text { Aucun } \\
\text { don } \\
0,00\end{array}$ & $\begin{array}{l}\text { Autre don } \\
\text { ailleurs } \\
1,00\end{array}$ & $\begin{array}{l}\text { Nc. } \\
\text { Autre } \\
\text { don } \\
2,00\end{array}$ & $\begin{array}{l}\text { Nc ail + } \\
\text { L. orig. } \\
3,00\end{array}$ & $\begin{array}{l}\text { Nc. } \\
+ \text { autre } \\
\text { don } \\
4,00\end{array}$ & $\begin{array}{l}\text { Nc } \\
\text { I.O } \\
5,00\end{array}$ & $\begin{array}{l}\text { Nc Dakar }+ \\
\text { autre don } \\
6,00\end{array}$ & $\begin{array}{l}\text { Nc } \\
\text { Dakar } \\
\text { row } \\
7,00\end{array}$ & $\begin{array}{l}\text { TOT } \\
\text { AL }\end{array}$ \\
\hline $\begin{array}{l}25-34 \\
\text { ans }\end{array}$ & $\begin{array}{l}71 \\
32,1 \%\end{array}$ & $\begin{array}{l}120 \\
54,3 \%\end{array}$ & $\begin{array}{l}5 \\
2,3 \%\end{array}$ & $\begin{array}{l}1 \\
0,5 \%\end{array}$ & $\begin{array}{l}14 \\
6,3 \%\end{array}$ & $\begin{array}{l}9 \\
4,1 \\
\%\end{array}$ & & $\begin{array}{l}1 \\
0,5 \%\end{array}$ & 221 \\
\hline $\begin{array}{l}35-44 \\
\text { ans }\end{array}$ & $\begin{array}{l}33 \\
14,5 \%\end{array}$ & $\begin{array}{l}148 \\
64,9 \%\end{array}$ & $\begin{array}{l}6 \\
2,6 \%\end{array}$ & $\begin{array}{l}3 \\
1,3 \%\end{array}$ & $\begin{array}{l}19 \\
8,3 \%\end{array}$ & $\begin{array}{l}6 \\
2,6 \\
\%\end{array}$ & $\begin{array}{l}7 \\
3,1 \%\end{array}$ & $\begin{array}{l}6 \\
2,6 \%\end{array}$ & 228 \\
\hline $\begin{array}{l}45-59 \\
\text { ans }\end{array}$ & $\begin{array}{l}84 \\
32,3 \%\end{array}$ & $\begin{array}{l}137 \\
52,7 \%\end{array}$ & $\begin{array}{l}6 \\
2,3 \%\end{array}$ & $\begin{array}{l}3 \\
1,2 \%\end{array}$ & $\begin{array}{l}16 \\
6,2 \%\end{array}$ & $\begin{array}{l}5 \\
1,9 \\
\%\end{array}$ & $\begin{array}{l}6 \\
2,3 \%\end{array}$ & $\begin{array}{l}3 \\
1,2 \%\end{array}$ & 260 \\
\hline
\end{tabular}

Tableau 5 : Don au père ou à la mère par génération. Hommes nés à Dakar

\begin{tabular}{|c|c|c|c|c|c|c|c|c|}
\hline & \multicolumn{8}{|c|}{ Destinataires } \\
\hline Générations & $\begin{array}{l}\text { Aucun } \\
\text { don } \\
0,00\end{array}$ & $\begin{array}{l}\begin{array}{l}\text { Autre } \\
\text { don }\end{array} \\
1,00\end{array}$ & $\begin{array}{l}\text { PM } \\
\text { aill. } \\
2,00\end{array}$ & $\begin{array}{l}\text { pm ail + } \\
\text { autre don } \\
3,00\end{array}$ & $\begin{array}{l}\text { pm } 1 \\
\text { ori } \\
\text { L orig } \\
4,00\end{array}$ & $\begin{array}{l}\text { pm } \\
\text { Dakar } \\
6,00\end{array}$ & $\begin{array}{l}\text { pm Dakar + } \\
\text { aut do } \\
7,00\end{array}$ & $\begin{array}{l}\text { Row } \\
\text { TOTAL }\end{array}$ \\
\hline
\end{tabular}




\begin{tabular}{|c|c|c|c|c|c|c|c|c|}
\hline $25-34$ ans & $\begin{array}{l}98 \\
59,0 \%\end{array}$ & $\begin{array}{l}51 \\
30,7 \%\end{array}$ & $\begin{array}{l}3 \\
1,8 \%\end{array}$ & $\begin{array}{l}1 \\
0,6 \%\end{array}$ & $\begin{array}{l}1 \\
0,6 \%\end{array}$ & $\begin{array}{l}6 \\
3,6 \%\end{array}$ & $\begin{array}{l}6 \\
3,6 \%\end{array}$ & 166 \\
\hline $35-44$ ans & $\begin{array}{l}30 \\
27,5 \%\end{array}$ & $\begin{array}{l}54 \\
49,5 \%\end{array}$ & $\begin{array}{l}1 \\
0,9 \%\end{array}$ & $\begin{array}{l}1 \\
0,9 \%\end{array}$ & & $\begin{array}{l}13 \\
11,9 \%\end{array}$ & $\begin{array}{l}10 \\
9,2 \%\end{array}$ & 109 \\
\hline 45-59 ans & $\begin{array}{l}18 \\
28,1 \%\end{array}$ & $\begin{array}{l}25 \\
39,1 \%\end{array}$ & & & & $\begin{array}{l}15 \\
23,4 \%\end{array}$ & $\begin{array}{l}6 \\
9,4 \%\end{array}$ & 64 \\
\hline
\end{tabular}

Tableau 6 : Don au père ou à la mère par génération. Hommes migrants

\begin{tabular}{|c|c|c|c|c|c|c|c|c|c|}
\hline & Destin & ires & & & & & & & \\
\hline $\begin{array}{l}\text { Générat } \\
\text { ions }\end{array}$ & $\begin{array}{l}\text { Aucun } \\
\text { don } \\
0,00\end{array}$ & $\begin{array}{l}\text { Autre } \\
\text { don } \\
1,00\end{array}$ & $\begin{array}{l}\mathrm{PM} \\
\text { aill. } \\
2,00\end{array}$ & $\begin{array}{l}\text { pm ail + } \\
\text { autre don } \\
3,00\end{array}$ & $\begin{array}{l}\text { pm } 1 \\
\text { ori } \\
\text { L orig } \\
4,00\end{array}$ & $\begin{array}{l}\text { Pm 1.o + } \\
\text { aut don } \\
5,00\end{array}$ & $\begin{array}{l}\text { pm } \\
\text { Dakar } \\
6,00\end{array}$ & $\begin{array}{l}\text { pm Dakar } \\
+ \text { aut do } \\
7,00\end{array}$ & $\begin{array}{l}\text { Row } \\
\text { TOT } \\
\text { AL }\end{array}$ \\
\hline $\begin{array}{l}25-34 \\
\text { ans }\end{array}$ & $\begin{array}{l}71 \\
32,1 \%\end{array}$ & $\begin{array}{l}55 \\
24,9 \%\end{array}$ & $\begin{array}{l}10 \\
4,5 \%\end{array}$ & $\begin{array}{l}4 \\
1,8 \%\end{array}$ & $\begin{array}{l}51 \\
23,1 \%\end{array}$ & $\begin{array}{l}24 \\
10,9 \%\end{array}$ & $\begin{array}{l}5 \\
2,3 \%\end{array}$ & $\begin{array}{l}1 \\
0,5 \%\end{array}$ & \\
\hline $\begin{array}{l}35-44 \\
\text { ans }\end{array}$ & $\begin{array}{l}33 \\
14,5 \%\end{array}$ & $\begin{array}{l}95 \\
41,7 \%\end{array}$ & $\begin{array}{l}6 \\
2,6 \%\end{array}$ & $\begin{array}{l}5 \\
2,2 \%\end{array}$ & $\begin{array}{l}57 \\
25,0 \%\end{array}$ & $\begin{array}{l}21 \\
9,2 \%\end{array}$ & $\begin{array}{l}6 \\
2,6 \%\end{array}$ & $\begin{array}{l}5 \\
2,2 \%\end{array}$ & 228 \\
\hline $\begin{array}{l}45-59 \\
\text { ans }\end{array}$ & $\begin{array}{l}84 \\
32,3 \%\end{array}$ & $\begin{array}{l}115 \\
4,2 \%\end{array}$ & $\begin{array}{l}8 \\
3,1 \%\end{array}$ & $\begin{array}{l}5 \\
1,9 \%\end{array}$ & $\begin{array}{l}26 \\
10 \%\end{array}$ & $\begin{array}{l}13 \\
5 \%\end{array}$ & $\begin{array}{l}7 \\
2,7 \%\end{array}$ & $\begin{array}{l}2 \\
0,8 \%\end{array}$ & 260 \\
\hline
\end{tabular}

Tableau 7 : Don au père ou à la mère par génération. Femmes nées à Dakar

\begin{tabular}{|c|c|c|c|c|c|c|c|}
\hline & \multicolumn{7}{|c|}{ Destinataires } \\
\hline Générations & $\begin{array}{l}\text { Aucun } \\
\text { don } \\
0,00\end{array}$ & $\begin{array}{l}\text { Autre } \\
\text { don } \\
1,00\end{array}$ & $\begin{array}{l}\mathrm{PM} \\
\text { aill. } \\
2,00\end{array}$ & $\begin{array}{l}\text { pm } 10+\text { aut } \\
\text { don } \\
5,00\end{array}$ & $\begin{array}{l}\text { pm } \\
\text { Dakar } \\
6,00\end{array}$ & $\begin{array}{l}\text { pm Dakar + aut } \\
\text { do } \\
7,00\end{array}$ & $\begin{array}{l}\text { Row } \\
\text { TOTAL }\end{array}$ \\
\hline $25-34$ ans & $\begin{array}{l}51 \\
63 \%\end{array}$ & $\begin{array}{l}15 \\
18,5 \%\end{array}$ & & & $\begin{array}{l}9 \\
11,1 \%\end{array}$ & $\begin{array}{l}6 \\
7,4 \%\end{array}$ & 81 \\
\hline $35-44$ ans & $\begin{array}{l}26 \\
52 \%\end{array}$ & $\begin{array}{l}19 \\
38 \%\end{array}$ & $\begin{array}{l}1 \\
2,0 \%\end{array}$ & $\begin{array}{l}1 \\
2,0 \%\end{array}$ & $\begin{array}{l}2 \\
4,0 \%\end{array}$ & $\begin{array}{l}1 \\
2,0 \%\end{array}$ & 50 \\
\hline $45-59$ ans & $\begin{array}{l}16 \\
59,3 \%\end{array}$ & $\begin{array}{l}7 \\
25,9 \%\end{array}$ & $\begin{array}{l}2 \\
7,4 \%\end{array}$ & & $\begin{array}{l}2 \\
7,4 \%\end{array}$ & & 27 \\
\hline
\end{tabular}


Tableau 8 : Don au père ou à la mère par génération. Femmes migrantes

\begin{tabular}{|c|c|c|c|c|c|c|c|c|c|}
\hline & Destina & ires & & & & & & & \\
\hline $\begin{array}{l}\text { Générat } \\
\text { ions }\end{array}$ & $\begin{array}{l}\text { Aucun } \\
\text { don } \\
0,00\end{array}$ & $\begin{array}{l}\text { Autre } \\
\text { don } \\
1,00\end{array}$ & $\begin{array}{l}\mathrm{PM} \\
\text { aill. } \\
2,00\end{array}$ & $\begin{array}{l}\text { pm ail + } \\
\text { autre don } \\
3,00\end{array}$ & $\begin{array}{l}\text { pm } 1 \\
\text { ori } \\
\text { L orig } \\
4,00\end{array}$ & $\begin{array}{l}\text { Pm } 1.0+ \\
\text { aut don } \\
5,00\end{array}$ & $\begin{array}{l}\text { pm } \\
\text { Dakar } \\
6,00\end{array}$ & $\begin{array}{l}\text { pm Dakar } \\
+ \text { aut do } \\
7,00\end{array}$ & $\begin{array}{l}\text { Row } \\
\text { TOT } \\
\text { AL }\end{array}$ \\
\hline $\begin{array}{l}25-34 \\
\text { ans }\end{array}$ & $\begin{array}{l}47 \\
52,8 \%\end{array}$ & $\begin{array}{l}14 \\
15,7 \%\end{array}$ & $\begin{array}{l}2 \\
2,2 \%\end{array}$ & & $\begin{array}{l}15 \\
16,9 \%\end{array}$ & $\begin{array}{l}5 \\
5,6 \%\end{array}$ & $\begin{array}{l}5 \\
5,6 \%\end{array}$ & $\begin{array}{l}1 \\
1,1 \%\end{array}$ & 89 \\
\hline $\begin{array}{l}35-44 \\
\text { ans }\end{array}$ & $\begin{array}{l}64 \\
56,6 \%\end{array}$ & $\begin{array}{l}19 \\
16,8 \%\end{array}$ & & $\begin{array}{l}4 \\
3,5 \%\end{array}$ & $\begin{array}{l}14 \\
12,4 \%\end{array}$ & $\begin{array}{l}6 \\
5,3 \%\end{array}$ & $\begin{array}{l}3 \\
2,7 \%\end{array}$ & $\begin{array}{l}3 \\
2,7 \%\end{array}$ & 113 \\
\hline $\begin{array}{l}45-59 \\
\text { ans }\end{array}$ & $\begin{array}{l}77 \\
57,9 \%\end{array}$ & $\begin{array}{l}40 \\
30,1 \%\end{array}$ & $\begin{array}{l}3 \\
2,3 \%\end{array}$ & & $\begin{array}{l}7 \\
5,3 \%\end{array}$ & $\begin{array}{l}4 \\
3,0 \%\end{array}$ & & $\begin{array}{l}2 \\
1,5 \%\end{array}$ & 133 \\
\hline
\end{tabular}

Tableau 9 : Don à la parenté par génération. Hommes nés à Dakar

\begin{tabular}{|c|c|c|c|c|c|c|c|c|c|}
\hline & \multicolumn{9}{|c|}{ Destinataires } \\
\hline Générations & $\begin{array}{l}\begin{array}{l}\text { Aucun } \\
\text { don }\end{array} \\
0,00\end{array}$ & $\begin{array}{l}\begin{array}{l}\text { Autre } \\
\text { don }\end{array} \\
1,00\end{array}$ & $\begin{array}{l}\text { Par } \\
\text { aill. } \\
2,00\end{array}$ & $\mid \begin{array}{l}\text { Par ail }+ \\
\text { autre } \\
\text { don } \\
3,00\end{array}$ & $\begin{array}{l}\text { par } 1 \\
\text { orig } \\
4,00\end{array}$ & $\begin{array}{ll}\text { Par } & 1.0 \\
+ & \text { aut } \\
\text { don } & \\
5,00 & \end{array}$ & $\begin{array}{l}\text { Par } \\
\text { Dakar } \\
6,00\end{array}$ & $\begin{array}{l}\text { Par } \\
\text { Dakar }+ \\
\text { aut do } \\
7,00\end{array}$ & $\begin{array}{l}\text { Row } \\
\text { TOTAL }\end{array}$ \\
\hline $25-34$ ans & $\begin{array}{l}98 \\
59 \%\end{array}$ & $\begin{array}{l}38 \\
22,9 \%\end{array}$ & $\begin{array}{l}6 \\
3,6 \%\end{array}$ & $\begin{array}{l}1 \\
0,6 \%\end{array}$ & & $\begin{array}{l}1 \\
0,6 \%\end{array}$ & $\begin{array}{l}13 \\
7,8 \%\end{array}$ & $\begin{array}{l}9 \\
5,4 \%\end{array}$ & 166 \\
\hline $35-44$ ans & $\begin{array}{l}30 \\
27,5 \%\end{array}$ & $\begin{array}{l}43 \\
39,4 \%\end{array}$ & $\begin{array}{l}6 \\
5,5 \%\end{array}$ & $\begin{array}{l}1 \\
0,9 \%\end{array}$ & $\begin{array}{l}2 \\
1,8 \%\end{array}$ & & $\begin{array}{l}14 \\
12,8 \%\end{array}$ & $\begin{array}{l}13 \\
11,9 \%\end{array}$ & 109 \\
\hline 45-59 ans & $\begin{array}{l}18 \\
28,1 \%\end{array}$ & $\begin{array}{l}29 \\
45,3 \%\end{array}$ & $\begin{array}{l}2 \\
3,1 \%\end{array}$ & & $\begin{array}{l}1 \\
1,6 \%\end{array}$ & & $\begin{array}{l}7 \\
10,9 \%\end{array}$ & $\begin{array}{l}7 \\
10,9 \%\end{array}$ & 64 \\
\hline
\end{tabular}

Tableau 10 : don à la parenté par génération. Hommes migrants 


\begin{tabular}{|c|c|c|c|c|c|c|c|c|c|}
\hline Générations & $\begin{array}{l}\begin{array}{l}\text { Aucun } \\
\text { don }\end{array} \\
0,00\end{array}$ & $\begin{array}{l}\text { Autre } \\
\text { ail. } \\
1,00\end{array}$ & $\begin{array}{ll}\text { Par } & + \\
\text { aut } & \\
\text { don } & \\
2,00 & \end{array}$ & $\begin{array}{l}\text { Par ail } \\
\text { l orig. } \\
3,00\end{array}$ & $\begin{array}{l}\text { par }+ \\
\text { aut don } \\
4,00\end{array}$ & $\begin{array}{l}\text { Par 1.o } \\
\text { Dakar } \\
5,00\end{array}$ & $\mid \begin{array}{ll}\text { Par } & r+ \\
\text { aut } & \\
\text { don } & \\
6,00 & \end{array}$ & $\begin{array}{l}\text { Par } \\
\text { Dakar } \\
7,00\end{array}$ & $\begin{array}{l}\text { Row } \\
\text { TOTAL }\end{array}$ \\
\hline $25-34$ ans & $\begin{array}{l}71 \\
32,1 \%\end{array}$ & $\begin{array}{l}109 \\
49,3 \%\end{array}$ & $\begin{array}{l}6 \\
2,7 \%\end{array}$ & $\begin{array}{l}6 \\
2,7 \%\end{array}$ & $\begin{array}{l}14 \\
6,3 \%\end{array}$ & $\begin{array}{l}7 \\
3,2 \%\end{array}$ & $\begin{array}{l}3 \\
1,4 \%\end{array}$ & $\begin{array}{l}5 \\
2,3 \%\end{array}$ & 221 \\
\hline $35-44$ ans & $\begin{array}{l}33 \\
14,5 \%\end{array}$ & $\begin{array}{l}126 \\
55,3 \%\end{array}$ & $\begin{array}{l}5 \\
2,2 \%\end{array}$ & $\begin{array}{l}5 \\
2,2 \%\end{array}$ & $\begin{array}{l}16 \\
7,0 \%\end{array}$ & $\begin{array}{l}14 \\
6,1 \%\end{array}$ & $\begin{array}{l}15 \\
6,6 \%\end{array}$ & $\begin{array}{l}14 \\
6,1 \%\end{array}$ & 228 \\
\hline $45-59$ ans & $\begin{array}{l}84 \\
32,3 \%\end{array}$ & $\begin{array}{l}91 \\
35,0 \%\end{array}$ & $\begin{array}{l}9 \\
3,5 \%\end{array}$ & $\begin{array}{l}5 \\
1,9 \%\end{array}$ & $\begin{array}{l}32 \\
12,3 \%\end{array}$ & $\begin{array}{l}18 \\
6,9 \%\end{array}$ & $\begin{array}{l}12 \\
4,6 \%\end{array}$ & $\begin{array}{l}9 \\
3,5 \%\end{array}$ & 260 \\
\hline
\end{tabular}

Tableau 11 : Soutien financier reçu d'autres personnes selon l'âge du bénéficiaire. Sexe masculin

\begin{tabular}{|c|c|c|c|c|c|c|c|}
\hline $\begin{array}{l}\text { Groupe } \\
\text { d'âge }\end{array}$ & Statut & $\begin{array}{l}\text { Aucun } \\
\text { soutien }\end{array}$ & $\begin{array}{l}\text { Soutien du } \\
\text { noyau } \\
\text { conjugal }\end{array}$ & $\begin{array}{l}\text { Soutien père/ } \\
\text { mère ou } \\
\text { parent }\end{array}$ & $\begin{array}{l}\text { Soutien } \\
\text { frère sœur }\end{array}$ & Autre & Assocation \\
\hline $\begin{array}{l}23-34 \\
\text { ans }\end{array}$ & $\begin{array}{l}\text { Né à } \\
\text { Dakar } \\
\text { Migrant }\end{array}$ & $\begin{array}{l}80,1 \\
80,1\end{array}$ & 0,6 & $\begin{array}{l}6,6 \\
5,9\end{array}$ & $\begin{array}{l}6,6 \\
9,9\end{array}$ & $\begin{array}{l}6,0 \\
4,5\end{array}$ & $\begin{array}{l}0,6 \\
1,3\end{array}$ \\
\hline $\begin{array}{l}35-44 \\
\text { ans }\end{array}$ & $\begin{array}{l}\text { Né à } \\
\text { Dakar } \\
\text { Migrant }\end{array}$ & $\begin{array}{l}87,2 \\
88,2\end{array}$ & & $\begin{array}{l}1,8 \\
3,9\end{array}$ & $\begin{array}{l}7,4 \\
3,9\end{array}$ & $\begin{array}{l}2,8 \\
1,8\end{array}$ & $\begin{array}{l}0,9 \\
2,2\end{array}$ \\
\hline $\begin{array}{l}45-59 \\
\text { ans }\end{array}$ & $\begin{array}{l}\text { Né à } \\
\text { Dakar } \\
\text { Migrant }\end{array}$ & $\begin{array}{l}82,8 \\
83,1\end{array}$ & $\begin{array}{l}6,3 \\
3,1\end{array}$ & 1,9 & $\begin{array}{l}4,7 \\
5,4\end{array}$ & $\begin{array}{l}6,3 \\
6,5\end{array}$ & 0,8 \\
\hline
\end{tabular}

Tableau 12 : Soutien financier reçu d'autres personnes selon l'âge du bénéficiaire. Sexe féminin

\begin{tabular}{|c|c|c|c|c|c|c|c|}
\hline $\begin{array}{l}\text { Groupe } \\
\text { d'âge }\end{array}$ & Statut & $\begin{array}{l}\text { Aucun } \\
\text { soutien }\end{array}$ & $\begin{array}{l}\text { Soutien du } \\
\text { noyau } \\
\text { conjugal }\end{array}$ & 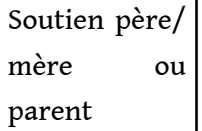 & $\begin{array}{l}\text { Soutien } \\
\text { frère sœur }\end{array}$ & Autre & Assocation \\
\hline $\begin{array}{l}23-34 \\
\text { ans }\end{array}$ & $\begin{array}{l}\text { Né à } \\
\text { Dakar } \\
\text { Migrant }\end{array}$ & $\begin{array}{l}56.8 \\
67,4\end{array}$ & $\begin{array}{l}11,1 \\
6,8\end{array}$ & $\begin{array}{l}9,9 \\
5,6\end{array}$ & $\begin{array}{l}6,2 \\
11,3\end{array}$ & $\begin{array}{l}16 \\
4,5\end{array}$ & $\begin{array}{l}7,4 \\
7,9\end{array}$ \\
\hline
\end{tabular}




\begin{tabular}{|c|c|c|c|c|c|c|c|}
\hline $\begin{array}{l}35-44 \\
\text { ans }\end{array}$ & $\begin{array}{l}\text { Né à } \\
\text { Dakar } \\
\text { Migrant }\end{array}$ & $\begin{array}{l}62,0 \\
65,5\end{array}$ & $\begin{array}{l}6,0 \\
6,2\end{array}$ & $\begin{array}{l}10,0 \\
5,3\end{array}$ & $\begin{array}{l}10,0 \\
13,3\end{array}$ & \begin{tabular}{|l}
2 \\
1,8
\end{tabular} & $\begin{array}{l}14 \\
9,7\end{array}$ \\
\hline $\begin{array}{l}45-59 \\
\text { ans }\end{array}$ & $\begin{array}{l}\text { Né à } \\
\text { Dakar } \\
\text { Migrant }\end{array}$ & $\begin{array}{l}63,0 \\
66,9\end{array}$ & $\begin{array}{r}29,6 \\
19,6\end{array}$ & 6,8 & $\begin{array}{l}3,7 \\
3,0\end{array}$ & $\begin{array}{l}3,7 \\
2,3\end{array}$ & $\begin{array}{l}3,7 \\
3,0\end{array}$ \\
\hline
\end{tabular}

Tableau 13 : Lien avec intermédiaire par génération pour l'accès au logement (migrants arrivés à Dakar après l'âge de 20 ans) pour tous les statuts résidentiels

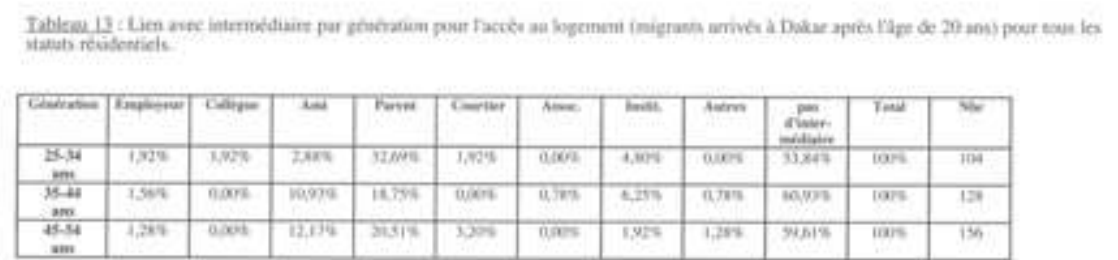

Voir tableau 13 en annexe

Tableau 14 : Lien des cohabitants (hébergés et colocataires) dans le premier logement à Dakar par génération (migrants arrivés à Dakar après l'âge de 20 ans)

\begin{tabular}{|l|l|l|l|l|l|l|l||l|}
\hline $\begin{array}{l}\text { Généra } \\
\text { tion }\end{array}$ & Epouse & $\begin{array}{l}\text { Ascendants } \\
\text { père/mère }\end{array}$ & $\begin{array}{l}\text { Colla } \\
\text { téraux }\end{array}$ & $\begin{array}{l}\text { Autres } \\
\text { parents }\end{array}$ & $\begin{array}{l}\text { Sans } \\
\text { lien }\end{array}$ & $\begin{array}{l}\text { Emp } \\
\text { loyeur }\end{array}$ & $\begin{array}{l}\text { To } \\
\text { tal }\end{array}$ & Nbr \\
\hline \hline $25-34$ ans & 0 & $41,33 \%$ & $28 \%$ & $14,67 \%$ & $5,33 \%$ & $10,67 \%$ & $100 \%$ & 75 \\
\hline $35-44$ ans & 0 & $25 \%$ & $28,75 \%$ & $22,50 \%$ & $11,25 \%$ & $12,50 \%$ & $100 \%$ & 80 \\
\hline \hline $45-54$ ans & $2,41 \%$ & $33,73 \%$ & $18,07 \%$ & $9,64 \%$ & $16,87 \%$ & $19,28 \%$ & $100 \%$ & 83 \\
\hline
\end{tabular}

Tableau 15 : Lien avec personnes-ressources par génération (répondants indépendants hommes nés hors de Dakar / Pikine, emplois juste arrivée à Dakar).

\begin{tabular}{|l|l||l|l|l|l||l|l|}
\hline $\begin{array}{l}\text { Généra } \\
\text { tion }\end{array}$ & $\begin{array}{l}\text { Pas } \\
\text { d'intermédiaire }\end{array}$ & $\begin{array}{l}\text { Epoux et } \\
\text { père }\end{array}$ & $\begin{array}{l}\text { Ascendant } \\
\text { et } \\
\text { collatéraux }\end{array}$ & $\begin{array}{l}\text { Autres } \\
\text { parents }\end{array}$ & $\begin{array}{l}\text { Sans lien } \\
\text { de parenté } \\
\text { ou } \\
\text { d'alliance }\end{array}$ & Total & Nbr \\
\hline \hline $25-34$ ans & 0 & $41,33 \%$ & $28 \%$ & $14,67 \%$ & $5,33 \%$ & $100 \%$ & 75 \\
\hline $35-44$ ans & 0 & $25 \%$ & $28,75 \%$ & $22,50 \%$ & $11,25 \%$ & $100 \%$ & 80 \\
\hline \hline $45-54$ ans & $2,41 \%$ & $23,73 \%$ & $18,07 \%$ & $9,64 \%$ & $16,87 \%$ & $100 \%$ & 83 \\
\hline
\end{tabular}


Tableau 16 : Indépendants hommes nés hors de Dakar / Pikine emploi après une période de chômage, d'apprentissage, d'étude ou d'inactivité

\begin{tabular}{|l|l||l|l|l|l|l|l|}
\hline $\begin{array}{l}\text { Généra } \\
\text { tion }\end{array}$ & $\begin{array}{l}\text { Pas } \\
\text { d'intermédiaire }\end{array}$ & $\begin{array}{l}\text { Père } / \\
\text { ascendant collatéraux }\end{array}$ & $\begin{array}{l}\text { Autres } \\
\text { parents- } \\
\text { parents }\end{array}$ & $\begin{array}{l}\text { Sans } \\
\text { lien }\end{array}$ & $\begin{array}{l}\text { Associa } \\
\text { tion }\end{array}$ & Total & Nbr \\
\hline \hline $25-34$ ans & $64,71 \%$ & $26,47 \%$ & $2,94 \%$ & $2,94 \%$ & $2,94 \%$ & $100 \%$ & 34 \\
\hline $35-44$ ans & $74,07 \%$ & $14,81 \%$ & $3,70 \%$ & $7,41 \%$ & $0 \%$ & $100 \%$ & 27 \\
\hline \hline $45-54$ ans & $95 \%$ & $5 \%$ & $0 \%$ & $0 \%$ & $0 \%$ & $100 \%$ & 20 \\
\hline
\end{tabular}

Tableau 17 : Lien avec la personne-ressource par génération (Indépendants Hommes nés à Dakar / Pikine)

\begin{tabular}{|l|l||l||l|l||l|l|l|l|l|}
\hline $\begin{array}{l}\text { Généra } \\
\text { tion }\end{array}$ & $\begin{array}{l}\text { Pas } \\
\text { d'inter } \\
\text { médiaire }\end{array}$ & Fils & Père & $\begin{array}{l}\text { Ascen } \\
\text { dants }\end{array}$ & $\begin{array}{l}\text { Colla } \\
\text { téraux }\end{array}$ & $\begin{array}{l}\text { Autre } \\
\text { parent }\end{array}$ & $\begin{array}{l}\text { Sans } \\
\text { lien }\end{array}$ & $\begin{array}{l}\text { To } \\
\text { tal }\end{array}$ & Nbr \\
\hline \hline $25-34$ ans & $\begin{array}{l}57,14 \\
\%\end{array}$ & $0 \%$ & $26,53 \%$ & $4,08 \%$ & $6,12 \%$ & $0 \%$ & $6,12 \%$ & $\begin{array}{l}100 \\
\%\end{array}$ \\
\hline \hline $35-44$ ans & $\begin{array}{l}72,41 \\
\%\end{array}$ & $3,45 \%$ & $13,79 \%$ & $3,49 \%$ & $3,45 \%$ & $3,45 \%$ & $0 \%$ & 100 \\
$\%$
\end{tabular}

Tableau 18 : Lien avec la personne-ressource pour l'accès au travail salarié par génération (Hommes nés hors de Dakar/Pikine, emploi après une période de chômage, d'apprentissage, d'étude ou d'inactivité)

\begin{tabular}{|l|l|l|l|l|}
\hline Génération & Lien de parenté & Sans lien de parenté ou d'alliance & Total & N \\
\hline \hline $25-34$ ans & $66,67 \%$ & $33,33 \%$ & $100 \%$ & 21 \\
\hline \hline $35-44$ ans & $39,39 \%$ & $42,31 \%$ & $100 \%$ & 33 \\
\hline \hline $45-54$ ans & $57,69 \%$ & $42,31 \%$ & $100 \%$ & 26 \\
\hline
\end{tabular}

Tableau 19 : Lien avec la personne-ressource pour l'accès au travail salarié par génération (Hommes nés à Dakar)

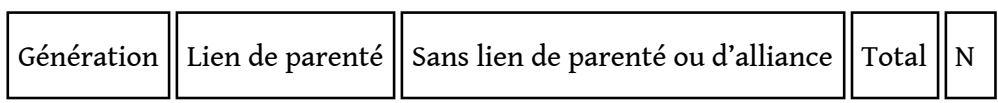




\begin{tabular}{|l|l|l|l|l|}
\hline $25-34$ ans & $63,33 \%$ & $36,67 \%$ & $100 \%$ & 30 \\
\hline \hline $35-44$ ans & $44,83 \%$ & $55,17 \%$ & $100 \%$ & 29 \\
\hline \hline $45-54$ ans & $38,46 \%$ & $61,54 \%$ & $100 \%$ & 13 \\
\hline
\end{tabular}

Tableau 20 : Recours à une personne-ressource pour l'accès au travail salarié (Hommes nés hors Dakar/Pikine, emploi jute après l'arrivée à Dakar)

\begin{tabular}{|l|l|l|l|l|}
\hline Génération & Intermédiaire & Pas d'intermédiaire & Total & $\mathrm{N}$ \\
\hline $25-34$ ans & $37,84 \%$ & $62,16 \%$ & $100 \%$ & 37 \\
\hline $35-44$ ans & $36,47 \%$ & $73,53 \%$ & $100 \%$ & 68 \\
\hline $45-54$ ans & $44,12 \%$ & $55,88 \%$ & $100 \%$ & 102 \\
\hline
\end{tabular}

Tableau 21 : Recours à une personne-ressource pour l'accès au travail salarié (Hommes nés hors Dakar/Pikine, emploi après période de chômage, d'apprentissage, d'étude ou d'inactivité)

\begin{tabular}{|l|l|l||c||c|}
\hline Génération & Intermédiaire & Pas d'intermédiaire & Total & N \\
\hline \hline $25-34$ ans & $42 \%$ & $58 \%$ & $100 \%$ & 50 \\
\hline \hline $35-44$ ans & $38,82 \%$ & $61,18 \%$ & $100 \%$ & 85 \\
\hline \hline $45-54$ ans & $31,33 \%$ & $68,67 \%$ & $100 \%$ & 83 \\
\hline
\end{tabular}

Tableau 22 : Recours à une personne-ressource pour l'accès au travail salarié (Hommes nés à Dakar/Pikine)

\begin{tabular}{|l|l|l||l|l|}
\hline Génération & Intermédiaire & Pas d'intermédiaire & Total & N \\
\hline \hline $25-34$ ans & $42,25 \%$ & $57,75 \%$ & $100 \%$ & 71 \\
\hline \hline $35-44$ ans & $37,66 \%$ & $62,34 \%$ & $100 \%$ & 77 \\
\hline $45-54$ ans & $28,89 \%$ & $71,11 \%$ & $100 \%$ & 45 \\
\hline
\end{tabular}

Tableau 23 : Lieu de travail personne-ressource pour accès au travail salarié par génération (Hommes nés hors de Dakar/Pikine, emploi juste après leur arrivée à Dakar)

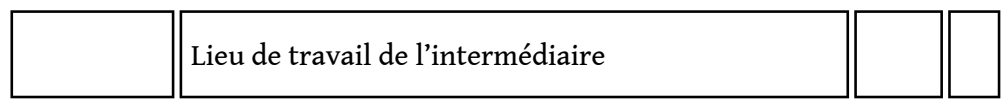




\begin{tabular}{|l|l|l|l|l|l|}
\hline Génération & Même entreprise que répondants & Hors entreprise & Total & $\mathrm{N}$ \\
\hline \hline $25-34$ ans & $57,14 \%$ & $42,86 \%$ & $100 \%$ & 14 \\
\hline $35-44$ ans & $61,11 \%$ & $38,89 \%$ & $100 \%$ & 18 \\
\hline $45-54$ ans & $64,44 \%$ & $35,56 \%$ & $100 \%$ & 45 \\
\hline
\end{tabular}

Tableau 24 : Lieu de travail personne-ressource pour accès au travail salarié par génération (Hommes nés à Dakar)

\begin{tabular}{|c|c|c|c|c|}
\hline & Lieu de travail de l'intermédiaire & & & \\
\hline Génération & Même entreprise que répondants & Hors entreprise & Total & $\mathrm{N}$ \\
\hline $25-34$ ans & $73,33 \%$ & $26,67 \%$ & $100 \%$ & 30 \\
\hline $35-44$ ans & $62,07 \%$ & $37,93 \%$ & $100 \%$ & 29 \\
\hline $45-54$ ans & $53,85 \%$ & $46,15 \%$ & $100 \%$ & 13 \\
\hline
\end{tabular}

Tableau 25 : Lieu de résidence de la personne-ressource pour accès emploi salarié par génération (Hommes nés hors de Dakar/Pikine, emploi après période de chômage, d'apprentissage, d'étude ou d'inactivité)

\begin{tabular}{|l|l|l|l|l|}
\hline & Lieu de travail de l'intermédiaire & & \\
\hline \hline Génération & Même logement & Hors logement & Total & $\mathrm{N}$ \\
\hline \hline $25-34$ ans & $42,86 \%$ & $57,15 \%$ & $100 \%$ & 14 \\
\hline \hline $35-44$ ans & $38,89 \%$ & $61,11 \%$ & $100 \%$ & 18 \\
\hline \hline $45-54$ ans & $28,89 \%$ & $71,11 \%$ & $100 \%$ & 45 \\
\hline
\end{tabular}

Tableau 26 : Lieu de résidence de la personne-ressource pour accès emploi salarié par génération (Hommes nés hors de Dakar/Pikine, emploi après période d'apprentissage, d'étude ou d'inactivité)

\begin{tabular}{|l|l|l|l|l|l|}
\hline & Lieu de travail de l'intermédiaire & \\
\hline Génération & Même logement & Hors logement & Total & \\
\hline $25-34$ ans & $23,81 \%$ & $76,19 \%$ & $100 \%$ & 21 \\
\hline \hline $35-44$ ans & $18,18 \%$ & $81,82 \%$ & $100 \%$ & 33 \\
\hline
\end{tabular}




\begin{tabular}{|l|l|l|l|}
\hline $45-54$ ans & $23,08 \%$ & $76,92 \%$ & $100 \%$ \\
\hline
\end{tabular}

Tableau 27 : Lieu de résidence de la personne-ressource pour accès emploi salarié par génération (Hommes nés à Dakar/Pikine)

\begin{tabular}{|l|l|l|l|l|l|}
\hline & Lieu de travail de l'intermédiaire & & \\
\hline \hline Génération & Même logement & Hors logement & Total & $\mathrm{N}$ \\
\hline $25-34$ ans & $30 \%$ & $70 \%$ & $100 \%$ & 30 \\
\hline $35-44$ ans & $27,59 \%$ & $72,42 \%$ & $100 \%$ & 29 \\
\hline \hline $45-54$ ans & $30,77 \%$ & $69,23 \%$ & $100 \%$ & 13 \\
\hline
\end{tabular}

Tableau 28 : Lieu de résidence de la personne-ressource pour accès emploi salarié par génération (Hommes nés hors de Dakar/Pikine, emploi après période de chômage, d'apprentissage, d'étude ou d'inactivité)

\begin{tabular}{|c|c|c|c|c|}
\hline & Lieu de travail de l'intermédiaire & & & \\
\hline Génération & Même entreprise que répondants & Hors entreprise & Total & $\mathrm{N}$ \\
\hline $25-34$ ans & $47,62 \%$ & $52,38 \%$ & $100 \%$ & 21 \\
\hline $35-44$ ans & $60,61 \%$ & $39,39 \%$ & $100 \%$ & 33 \\
\hline $45-54$ ans & $61,54 \%$ & $38,46 \%$ & $100 \%$ & 26 \\
\hline
\end{tabular}

\section{BIBLIOGRAPHIE}

ANTOINE Ph., BOCQUIER Ph., BARBARY O., FALL A.S., GUISSE Y ., NANITELAMIO J., 1992, L'insertion urbaine : le cas de Dakar, compte rendu d'une recherche financée par le Ministère de la Recherche et de la Technologie, IFAN et ORSTOM, $230 \mathrm{p}$.

ANTOINE Ph., 1991, Croissance urbaine et insertion des migrants dans les villes africaines. L'exemple de Dakar, in : Pratiques sociales et travail en milieu urbain, Les Cahiers n 16, ORSTOM/ SUD, Paris, p. 9-23.

ANTOINE Ph., SAVANE L., 1990, Urbanisation et migration en Afrique in : "The role of migration in African development : issues and policies for the 90s", UEPA, Conférence de Nairobi, p.55-81. 
BOCQUIER Ph., 1991, Les mutations du marché de l'emploi dans les pays en voie de développement : le cas d'une ville ouest-africaine, Dakar, in : Les Cahiers "Villes en Développement" $\mathrm{n}^{\circ}$ 11-91 Groupe inter-universitaire de Montréal, $32 \mathrm{p}$.

BOCQUIER Ph., FALL A.S., 1992, Le recours aux réseaux sociaux pour l'accès à l'emploi, le cas d'une ville en développement : Dakar, Communication à la 21ème Conférence de l'Association Canadienne des Etudes Africaines, Montréal, 13-16 mai 1992,24 p.

DIOP A.B., 1992, Les paysans du bassin arachidier. Conditions de vie et comportements de survie, in : Politique Africaine, n45, "Sénégal, La démocratie à l'épreuve", Karthala, p. 39-61.

DIOP M.C., 1982, Le phénomène associatif mouride en ville : expression du dynamisme confrérique, in : Psychopathologie Africaine, vol. XVIII, 3, p. 293-317.

DIOP M., 1989, Un exemple de non insertion urbaine : le cas des migrantes saisonnières de Basse Casamance à Dakar, in : L'insertion urbaine des migrants en Afrique, Editions de l'ORSTOM, (colloques et séminaires), p.79-89.

DUBRESSON A., 1990, Migrations, villes et villages de Côte-d'Ivoire, in : "The role of migration in African development : issues and policies for the 90s" UEPA, Commissioned papers, Nairobi, p. 96-108.

ELOUNDOU - ENYEGUE P.M., 1992, Solidarité dans la crise ou crise des solidarités familiales.

L'évolution post-1987 des échanges ville/campagne à Bafou et Yemessoa, communication au séminaire international sur "la crise et l'ajustement dans le milieu rural du Cameroun" Projet OCISCA, 10 p. multigr.

FALL A.S., 1991 (a), Réseaux de sociabilité et insertion urbaine dans l'agglomération de Dakar, Thèse de Doctorat de troisième cycle de Sociologie, Université Cheikh-Anta Diop, Dakar, 280 p. + annexes $420 \mathrm{p}$.

FALL A.S., 1991 (b), Une autre "famille" ? Les réseaux féminins de voisinage en ville, in : Conférence "Femme, Famille et Population", Burkina-Faso, 24-29 avril, par l'Union pour l'Etude de la Population Africaine, volume 2, p. 54-67

FALL A.S., 1991 (c), Du questionnaire biographique quantitatif aux entretiens approfondis sur les réseaux de sociabilité en ville, in : Pratiques sociales et travail en milieu urbain, Les Cahiers $n^{\circ} 14$, ORSTOM/SUD, Paris, p.37-50.

FALL A.S., 1991 (d), Le local ou le cosmopolite : Quel terrain d'études des réseaux de sociabilité en ville? in : Pratiques sociales et travail en milieu urbain, Les Cahiers n¹6, ORSTOM/SUD, Paris, $p$. 35-49.

FALL A.S., 1992, Une réponse à la crise de l'agriculture, la migration des Sereer du Siin (Sénégal) in : Société, Espace, Temps, 1, 1, nº sur "la crise de l'agriculture africaine", p. 138-149.

NDIAYE A.M.I., 1989, Les associations dans l'entreprise industrielle : le cas des daayire du Port Autonome de Dakar, Mémoire de maîtrise, dpt. de philosophie, Université Cheikh-Anta Diop de Dakar, 151 p. multigr.

O'NDEYE M., 1985, Les associations en villes africaines, Dakar et Brazzaville, L'Harmattan (Villes et Entreprises), $125 \mathrm{p}$.

PONTIE G., LERICOLLAIS A., 1991, Relations à distance des migrants sereer, Communication au séminaire IFAN-ORSTOM "Processus d'insertion urbaine et itinéraires résidentiels, professionnels et familiaux", 27 au 30 mai, Sally-Portudal (Sénégal), 29 p. multigr.

SINOU A., 1990, Dakar, Bulletin d'informations architecturales, Institut Français d'Architecture, supplément au n 141, été 1990. 
VALETTE A., 1990, Emploi et nouvelle politique industrielle au Sénégal, in : Pratiques sociales et travail en milieu urbain, Les Cahiers, $n^{\circ}$ spécial : Ouvriers, emploi et entreprises au Sénégal, ORSTOM/SUD, Paris, p. 83-96.

WERNER J. F., 1991, Urbanisation et déviance au Sénégal, Ph D. d'Anthropologie, Université de Montréal, $427 \mathrm{p}$.

ZAROUR CH., 1991, Étude du secteur informel de Dakar et de ses environs, Phase 3, Rapport final U.S.A.I.D. / Sénégal, 198 p.

\section{NOTES}

1.Cet article a fait l'objet d'une communication aux Journées de Montpellier de octobre 1992, mais sa problématique particulière nous a conduit à le publier dans cette rubrique (note de l'éditeur).

\section{AUTEUR}

\section{ABDOU SALAM FALL}

Dakar 